\title{
Régime variable dans un canal d'amenée associé à une galerie en charge
}

\section{Unsteady flow in a head race canal leading to a pressure tunnel}

\author{
L. ESCANIE \\ MEMinE DE L'INSTITUT, \\ INGÉNIEUR T.E.T. \\ DIRECTEuR de L'ÉCOLE NATIONALE SUPÉRIEURe \\ D'ÉLECTROTECHNIQUE ET D'HYDRAULIQUE DE TOULOUSE
}

PAR

$\mathrm{ET}$

\author{
J. NOUGARO \\ LAURÉAT DE L'INSTITUT \\ INGÉNIEUR H.E.T, \\ PROFESSEUR A L'ÉCOLE NATIONALE SUPÉrIEURE \\ D'ÉTECTROTECHNIQUE ET D'HYDRAULIQUUE DE TOULOUSE
}

Etude de deux problèmes associant les intumescences produites dans un canal d'amenée et les oscillations en masse engendrées dans une galerie en charge. - Le premier problème concerne un système galerie-cheminée d'équilibre faisant suite ḋ un canal da écoulement libre; le second problème concerne un siphon intercalé dans un canal d'amenée. - Présentation des méthodes d'etudes theoriques, analytiques ou graphiques ufilisees; concordance satisfaisante de leurs résultats avec les résultats expérimentaux obtenus.

\section{INTRODUCTION}

Dans le présent article, nous résumons les résultats que nous avons obtenus dans l'étude de deux problèmes associant les intumescences produites dans un canal d'amenée aux oscillations en masse engendrées dans une galerie en charge.

Le premier problème, exposé au chapitre 1 , concerne le fonctionnement d'une cheminée d'équilibre, dont le tunnel fait suite à un canal à écoulement libre.

La seconde question (chapitres II et III) envisage le cas d'un siphon intercalé dans un canal d'amenée.

Comme on le verra, la méthode de calcul utilisée pour le premier problème pent être facilement adaptée à l'étude du second phénomène. 


\section{CHAPITRE I}

\section{OSCILLATIONS DANS UNE CHEMINÉE D'ÉUILIBRE PLACÉE A L'AVAL D'UNE GALERIE EN CHARGE PRÉCÉdÉE ELLE-MÊME D'UN CANAL A ECOULEMENT LIBRE}

\section{1. - Méthode de calcul}

Nous allons considérer une installation d'amenée d'eau comportant, disposés en série, de l'amont vers l'aval :

- Un canal d'amenée à ćcoulement libre;

- Une galerie en charge;

-_ Une cheminée d'équilibre.

Lors d'un arrêt du débit des turbines, il se produit des oscillations du plan d'eau dans la cheminée d'équilibre et des intumescences dans le canal d'amenée.

Nous avons pu résoudre le problème des interactions entre les deux ouvrages au moyen d'un calcul aux différences finies dont nous allons exposer le principe.

Si l'on applique au système constitué par le canal d'amenée, la conduite en charge et la cheminée d'équilibre, l'équation générale des forces vives, on aboutit à la relation suivante :

$$
\frac{\mathrm{L}}{g f} \frac{d q}{d t}+Z^{\prime}{ }_{n}-Z^{\prime \prime}{ }_{n}+\mathrm{K} q^{2}=0
$$

Les notations sont indiquées sur la figure 1.

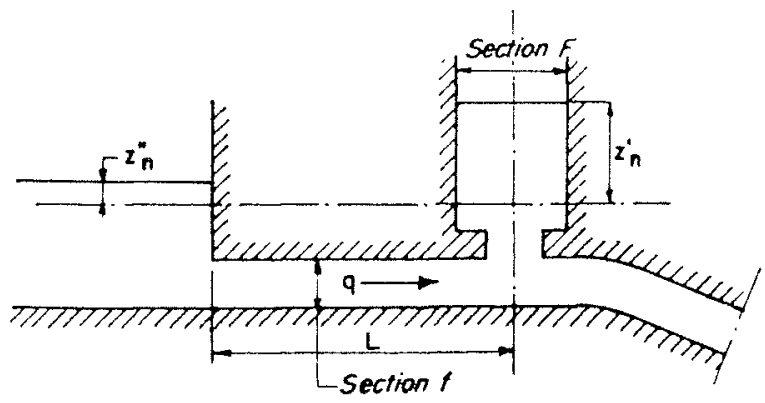

FIG. 1

En passant des différentielles aux différences finies et en considérant un intervalle de temps $\Delta t$, on peut en déduire la variation de débit $\Delta q$ produite dans la conduite en charge, durant cet intervalle.

$$
|\Delta q|=\frac{g f}{\mathrm{~L}} \Delta t\left[Z_{n}^{\prime}-Z_{n}^{\prime \prime}+\mathrm{K} q^{2}\right]
$$

Cette équation permet le calcul de $\Delta q$, à partir des valeurs moyennes de $Z_{n}^{\prime}, Z^{\prime \prime}{ }_{n}$ et $q$ durant l'intervalle considéré : il suffit, en pratique, de prendre pour celles-ci les valeurs obtenues à la fin de l'intervalle précédent.

On peut déduire, de cette variation de débit, la variation de hauteur d'eau dans la cheminée d'équilibre $\Delta Z^{\prime}$.

Simultanément, cette diminution de débit dans la galerie en charge provoque une surélévation de niveau au débouché aval du canal d'amenée.

Soit $\Delta q_{1}$ la variation du débit pendant le premier intervalle de temps $\Delta t$ et $\Delta Z_{1}^{\prime}$ la variation de hauteur d'eau dans la cheminée d'équilibre, on a :

$$
\Delta Z_{1}^{\prime}=\frac{Q_{0}-\left(\Delta q_{1} / 2\right)}{F^{2}} \cdot \Delta t
$$

On en déduit la hauteur d'eau $Z^{\prime}{ }_{1}$.

Durant le même intervalle de temps, le volume d'eau refoulé dans le canal d'amenée a pour valeur :

$$
\Delta \Omega_{1}=\Delta t \times \frac{\Delta q_{1}}{2}
$$

La surélévation du niveau $\Delta Z^{\prime \prime}{ }_{1}$ peut être calculée en écrivant que le volume d'eau qui passe dans l'intumescence élémentaire correspondante est égal au volume $\Delta \Omega_{1}$. On obtient ainsi :

$$
\begin{gathered}
1 / 2 \Delta Z^{\prime \prime}{ }_{1} c_{0} \cdot l_{0} \cdot \Delta t=\Delta t \frac{\Delta q_{1}}{2} \\
\Delta Z^{\prime \prime}{ }_{1}=\frac{\Delta q_{1}}{c_{0} l_{0}}
\end{gathered}
$$

et donc $Z^{\prime \prime}{ }_{1} ; c_{0}$ est la célérité de l'intumescence ainsi formée dans le canal d'amence et $l_{0}$ la largeur au miroir correspondante.

Durant le deuxième intervalle de temps $\Delta t$, en prenant les valeurs de $Z_{1}^{\prime}$ et $Z^{\prime \prime}{ }_{1}$ définies précédemment, on peut calculer $\Delta q_{2}$ et en déduire la variation de hauteur d'eau dans la cheminée d'équilibre :

$$
\Delta Z_{2}^{\prime}=\frac{Q_{0}-\Delta q_{1}-\left(\Delta q_{2} / 2\right)}{\mathrm{F}} . \Delta t
$$

et, par suite, la hauteur d'eau $Z_{2}^{\prime}$.

A la fin de cet intervalle de temps, le volume d'eau refoulé dans le canal, depuis l'instant initial, a pour valeur :

$$
\Delta \Omega_{2}=\Delta t\left[\Delta q_{1}+\frac{\Delta q_{2}}{2}\right]
$$


Si $\Delta Z^{\prime \prime}{ }_{2}$ est la surélévation correspondante, l'égalité des volumes permet d'écrire, comme précédemment :

$c_{1} \cdot \Delta t \cdot l_{1} \cdot \Delta Z^{\prime \prime}{ }_{1}+c_{1} \cdot \Delta t \cdot l_{1} \cdot \Delta Z^{\prime \prime}{ }_{2}=\Delta t\left[\Delta q_{1}+\Delta q_{2} / 2\right]$ soit :

$$
\Delta Z^{\prime \prime}{ }_{2}=\frac{1}{c_{1} l_{1}}\left(\Delta q_{1}+\frac{\Delta q_{2}}{2}\right)-\Delta Z^{\prime \prime}{ }_{1}
$$

en appelant $c_{1}$ la célérité de l'intumescence ainsi formée dans le canal d'amenée et $l_{1}$ la largeur au miroir correspondante.

On aurait de même, durant le troisième intervalle de temps $\Delta t$, en utilisant les valeurs de $Z_{2}^{\prime}$ et $Z^{\prime \prime}{ }_{2}$ calculées précédemment :

$$
\left|\Delta q_{3}\right|=\frac{g f}{L} \Delta t\left[Z_{2}^{\prime}-Z_{2}^{\prime \prime}+\mathrm{K} q_{2}{ }^{2}\right]
$$

On connaît ainsi la variation de hauteur d'eau dans la cheminée d'équilibre :

$$
\Delta Z_{3}^{\prime}=\frac{Q_{0}-\Delta q_{1}-\Delta q_{2}-\Delta q_{3} / 2}{F} \cdot \Delta t
$$

Le volume d'eau refoulé simultanément dans le canal a pour valeur :

$$
\Delta \Omega_{3}=\Delta t\left[\Delta q_{1}+\Delta q_{2}+\Delta q_{3} / 2\right]
$$

L'égalité des volumes d'eau permet d'aboutir à la valeur :

$\Delta Z^{\prime \prime}{ }_{3}=\frac{1}{c_{2} l_{2}}\left(\Delta q_{1}+\Delta q_{2}+\Delta q_{3} / 2\right)-\left(\Delta Z^{\prime \prime}{ }_{1}+\Delta Z^{\prime \prime}{ }_{2}\right)$

On opère ainsi pour les intervalles de temps successifs. En particulier, pendant le $n^{\text {ième }}$ intervalle $\Delta t$, on a :

$$
\begin{aligned}
\left|\Delta q_{n}\right| & =\frac{g f}{L} \Delta t\left[Z_{n-1}^{\prime}-Z^{\prime \prime}{ }_{n-1}+\mathrm{K} q^{2}{ }_{n-1}\right] \\
\Delta Z_{n}= & \frac{\mathrm{Q}_{0}-\Delta q_{1}--\Delta q_{2} \ldots \Delta q_{n-1}-\Delta q_{n} / 2}{\mathrm{~F}} \Delta t \\
\Delta Z^{\prime \prime}{ }_{n}= & \frac{1}{\mathrm{C}_{n-1} l_{n-1}}\left(\Delta q_{1}+\Delta q_{2}+\ldots+\frac{\Delta q_{n}}{2}\right) \\
& \quad-\left(\Delta Z^{\prime \prime}{ }_{1}+\Delta Z^{\prime \prime}{ }_{2}+\ldots+\Delta Z^{\prime \prime}{ }_{n-1}\right)
\end{aligned}
$$

\section{2. - Application à la cheminée d'équilibre de l'usine de Saint-Christaud}

La méthode exposée précédemment a été appliquée à l'étude des ouvrages d'amenée de l'usine de Saint-Christaud, en projet sur la Garonne.
Les données numériques retenues pour le calcul sont les suivantes :

$$
\begin{aligned}
\mathrm{Q}_{0} & =135 \mathrm{~m}^{3} / \mathrm{s} \\
\mathrm{F} & =154 \mathrm{~m}^{2} \\
\mathrm{~L} & =500 \mathrm{~m} \\
f & =38,5 \mathrm{~m}^{2} \\
\mathrm{P}_{0} & =0,61 \mathrm{~m} \\
\mathrm{R}_{0} & =9 \mathrm{~m}
\end{aligned}
$$

Cote du plan d'eau à l'entrée de la galerie (bassin de mise en charge) en régime permanent : 257,00 .

La méthode de calcul n'a été appliquée que pour la premic̀re oscillation du plan d'eau dans la cheminée d'équilibre.

Les comparaisons entre les résultats théoriques et des essais réalisés sur le modèle réduit de l'usine de Saint-Christaud ont porté, d'une part, sur la cheminée d'équilibre, d'autre part, sur le canal d'amenée.

La figure 2 reproduit la courbe d'oscillation déduite des calculs théoriques et la courbe d'oscillation enregistrée sur le modèle réduit, pour une fermeture du distributeur très brève ( $2 \mathrm{~s}$ en-

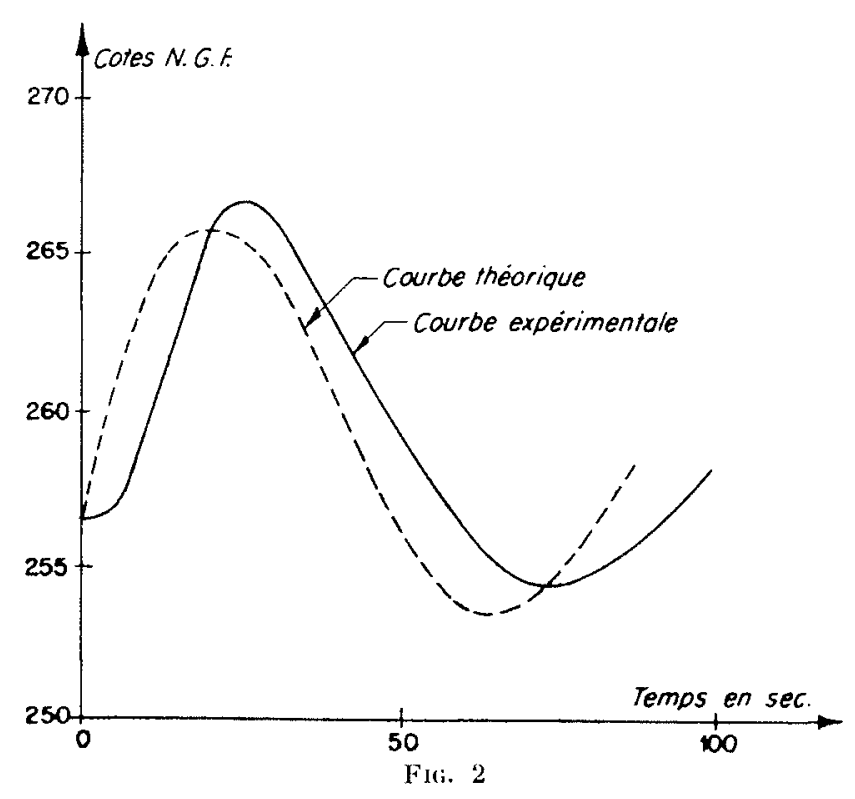

viron), que nous assimilons, pour les calculs théoriques, à une manœuvre instantanée, eu égard à la valeur $\mathrm{T}=84 \mathrm{~s}$ de la période du système galerie d'amenée-cheminée d'équilibre.

Les différences que l'on enregistre sont caractérisées par les valeurs suivantes :

- cote maximum théorique 266 ,

- cote maximum expérimentale 266,50,

- cote minimum théorique 253,50,

- cote minimum expérimentale 254,20. 


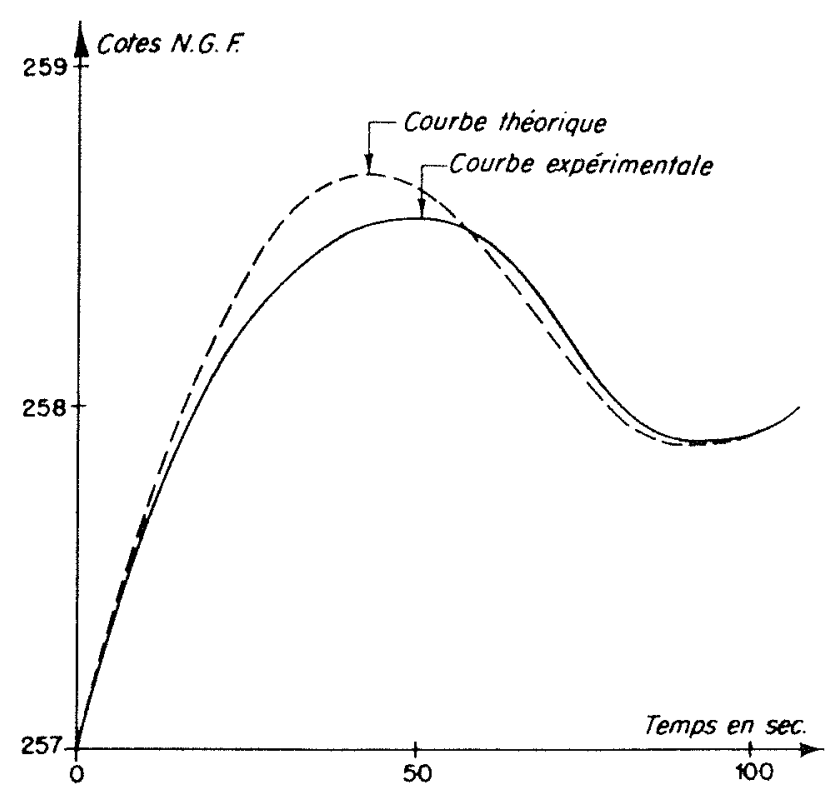

Fid. 3
On note la bonne approximation fournie par la méthode théorique que nous venons d'exposer.

Le maximum théorique est alteint au bout de $22,5 \mathrm{~s}$, alors que le maximum déduit de la courbe expérimentale est atteint au bout de 26 s. Le premier minimum théorique se situe à $68 \mathrm{~s}$ contre $74 \mathrm{~s}$, déduit de la courbe expérimentale.

La figure 3 reproduit la première oscillation d'intumescence au débouché de la galerie en charge, dans le canal d'amenée. Les deux courbes se rapportent respectivement aux résultats de l'étude théorique et à un enregistrement de coupure du débit réalisé sur le modèle réduit.

L'examen comparatif des deux courbes permet de se rendre compte de la bonne approximation obtenue; le maximum théorique situe la crête de la première oscillation à la cote 258,71 contre 258,58 fournie par la mesure expérimentale.

On voit que les résultats obtenus par notre méthode de calcul aux différences finies sont en bonne concordance avec les valeurs expérimentales.

\section{CHAPITRE II}

\section{GALERIE EN CHARGE SITUÉE ENTRE DEUX PARTIES D'UN CANAL D'AMENEE A ECOULEMENT LIBRE}

\section{1. - Généralités sur la méthode de calcul}

La méthode de calcul que nous avons exposée au chapitre précédent peut être étendue, moyennant quelques modifications, au cas d'une galerie en charge séparant deux parties d'un canal d'amenée à écoulement libre.

Si une intumescence prend naissance à la partie aval du canal d'amenée, elle se propage jusqu'au débouché du siphon. Le débit de celui-ci est alors progressivement réduit et une partie de l'onde incidente se transmet à la portion amont du canal d'amenée. L'équation d'oscillalion en masse s'applique à l'ensemble constitué par les deux portions du canal d'amenée et le siphon. On peut donc établir une méthode de calcul comparable à celle que nous avons indiquée au chapitre précédent. Mais, cette méthode, purement analytique, ne permet de calculer les variations de plan d'eau que dans les sections des canaux d'amenée placées immédiatement à l'amont et à l'aval du siphon.

Toutefois, en combinant ce calcul aux différences finies avec une méthode graphique d'étude des intumescences, on peut résoudre entièrement le problème et déterminer les intumescences sur toute la longueur du canal d'amenée

Nous allons exposer successivement les deux étapes du calcul.

\section{2. - Calcul aux différences finies}

En conservant les mêmes notations :

$q$ débit traversant le siphon,

$\mathrm{Q}_{0}$ débit maximum initial,

$Z_{n}^{\prime}$ hauteur d'eau à l'aval du siphon, comptée à partir du niveau amont,

$Z^{\prime \prime}{ }_{n}$ hauteur d'eau à l'amont, comptée à partir du niveau amont,

$L$ longueur du siphon,

$f$ section du siphon,

l'équation d'oscillation en masse s'écrit :

$$
\frac{\mathrm{L}}{g f} \frac{d q}{d t}+Z_{n}^{\prime}-Z^{\prime \prime}{ }_{n}+k q^{2}=0
$$

Les conditions initiales correspondent à l'instant où l'onde partie de l'extrémité aval du canal d'amenée atteint le débouché du siphon. Il se produit, à cet instant, une surélévation instantanée du plan d'eau due à l'onde incidente :

$$
\Delta Z_{0}^{\prime}=\frac{Q_{0}}{c_{0}^{\prime} l_{0}}
$$


$c_{0}^{\prime}$ étant la vitesse de propagation de l'intumescence, et

$l_{0}$ la largeur au miroir du canal.

Cette onde incidente se réfléchit en partie et donne lieu à une variation supplémentaire :

$$
\Delta \mathbf{Z}_{01}^{\prime}=\frac{\mathrm{Q}_{0}}{c_{01}^{\prime} l_{01}}
$$

$c^{\prime}{ }_{01}$ et $l_{01}$ étant les nouvelles valeurs de la vitesse de propagation et de la largeur au miroir.

Ces deux valeurs permettent ainsi de déterminer $Z^{\prime}$ à l'instant initial que l'on considère.

Le débit dans le siphon n'a subi aucune variation et, par suite, $Z^{\prime \prime}$ est encore nul.

On peut alors calculer, pendant un intervalle de temps $\Delta t$, la variation de débit $\Delta q_{1}$ qui se produit dans le siphon :

$$
\left|\Delta q_{1}\right|=\frac{g f}{\mathrm{~L}} \Delta t\left[Z^{\prime}{ }_{0}+k \mathbf{Q}^{2}{ }_{0}\right]
$$

Le débit moyen $Q_{0}-\left(\Delta q_{1} / 2\right)$ sortant du siphon provoque une surélévation à l'aval :

$$
\Delta Z_{1}^{\prime}=\frac{Q_{0}-\left(\Delta q_{1} / 2\right)}{c_{1}^{\prime} l_{1}^{\prime}}
$$

Simultanément, le débit moyen $\Delta q_{1} / 2$, qui ne pénètre plus à l'amont du siphon, crée une variation de niveau, à l'amont de ce dernier :

$$
\Delta Z^{\prime \prime}{ }_{1}=\frac{\Delta q_{1} / 2}{c^{\prime \prime}{ }_{1} l_{1}^{\prime \prime}}
$$

Connaissant ainsi à la fin du premier intervalle de temps les valeurs de $q_{1}, Z_{1}^{\prime}$ et $Z^{\prime \prime}{ }_{1}$, on peut calculer la nouvelle variation de débit $\Delta q_{2}$ durant le deuxième intervalle de temps $\Delta t$.

$$
\left|\Delta q_{2}\right|=\frac{g f}{\mathrm{~L}} \Delta t\left[Z_{1}^{\prime}-Z^{\prime \prime}{ }_{1}+k q_{1}{ }^{2}\right]
$$

Un débit $\mathrm{Q}_{0}-\Delta q_{1}-\left(\Delta q_{2} / 2\right)$ continuant à sortir du débouché aval du siphon conduit à une nouvelle surélévation :

$$
\Delta Z_{2}^{\prime}=\frac{\mathrm{Q}_{0}-\Delta q_{1}-\left(\Delta q_{2} / 2\right)}{c_{2}^{\prime} l_{2}^{\prime}}
$$

Simultanément, le débit qui ne pénètre plus à l'amont du siphon crée une variation de niveau durant cet intervalle :

$$
\Delta Z^{\prime \prime}{ }_{2}=\frac{\Delta q_{1}+\Delta q_{2} / 2}{c^{\prime \prime}{ }_{2} \nu_{2}^{\prime}}-\Delta Z^{\prime \prime}{ }_{1}
$$

On peut ainsi, pour tous les intervalles de temps successifs $\Delta t$, opérer pareillement et calculer à l'instant $n$ :

$$
\left|\Delta q_{n}\right|=\frac{g f}{\mathrm{~L}} \Delta t\left[Z_{n-1}^{\prime}-Z_{n-1}^{\prime \prime}+\mathrm{K} q_{n-1}^{2}\right]
$$

$$
\begin{array}{r}
\Delta Z_{n}^{\prime}=\frac{Q_{0}-\Delta q_{1}-\Delta q_{2} \ldots-\Delta q_{n-1}-\Delta q_{n} / 2}{c_{n}^{\prime} l^{\prime}{ }_{n}} \\
\Delta Z^{\prime \prime}=\frac{\Delta q_{1}+\Delta q_{2}+\ldots+\Delta q_{n-1}+\Delta q_{n} / 2}{c^{\prime \prime}{ }_{n} l^{\prime \prime}{ }_{n}} \\
-\left(\Delta Z^{\prime \prime}{ }_{1}+\ldots+\Delta Z^{\prime \prime}{ }_{n-1}\right)
\end{array}
$$

Ce calcul se poursuit jusqu'au moment où l'intumescence réfléchie à la chambre d'eau aval revient au siphon. Connaissant les éléments caractéristiques de cette onde, que l'on peut déterminer à partir des célérités et des largeurs du canal, il est possible de poursuivre le calcul selon la même méthode, en tenant compte des nouvelles conditions qui sont ainsi imposées.

\section{3. - Combinaison des méthodes de calcul analytiques et graphiques}

La méthode analytique exposée précédemment permet de relier les phénomènes de la partie aval du canal d'amenée à ceux de la partie amont. Cette méthode fournit ainsi des conditions aux limites se rapportant à l'aval et à l'amont $d u$ siphon.

On peut donc utiliser, pour les deux parties du canal d'amenée une des méthodes graphiques classiques permettant la détermination des surélévations engendrées par une intumescence.

Nous avons adopté la méthode simplifiée due à l'un de nous (1). Nous rappelons qu'elle repose sur la construction d'un diagramme en H.Q. utilisant des caractéristiques rectilignes de pente $(\alpha \pm U) / g s$ pour les ondes suivant le courant ou remontant ce dernier; elle est déduite de la méthode de Bergeron, dont elle permet, moyennant une approximation justifiée par de nombreuses expériences, de lever l'une des hypothèses restrictives.

Après la première réflexion d'intumescence en provenance de la chambre d'eau aval, l'équation d'oscillation en masse permet le caleul, à chaque instant, de la variation de débit du siphon, comme nous l'avons exposé précédemment :

$$
\left|\Delta q_{n}\right|=\frac{g f}{\mathrm{~L}} \Delta t\left[Z_{n-1}^{\prime}-Z_{n-1}^{\prime \prime}+k q_{n-1}^{2}\right]
$$

Le débit du siphon est, par suite :

$$
\mathrm{Q}_{0}-\Delta q_{1}-\Delta q_{2} \ldots-\frac{\Delta q_{n}}{2}
$$

On a ainsi un lieu du point de fonctionnement, à l’instant considéré, sur le graphique.

(1) J. Nougaro, Thèse de Doctorat, Faculté des Sciences, Toulouse, $n^{\circ} 457$ : Etude théorique et expérimentale de la propagation des intumescences dans les canaux découverts. 


\section{APPLICATION AU CAS D'UN SIPHON ÉTABLI SUR UN MODÈLE RÉdUIT DE CANAL D'AMENEE}

\section{Caractéristiques du système étudié}

Pour vérifier la méthode précédente, nous avons intercalé un siphon, sur un modèle réduit, à l'échelle $3 / 100$ du canal d'amenée de l'usine de Saint-Christaud, en couvrant, sur une certaine longueur, une partie du canal d'amenée par des dalles de béton.

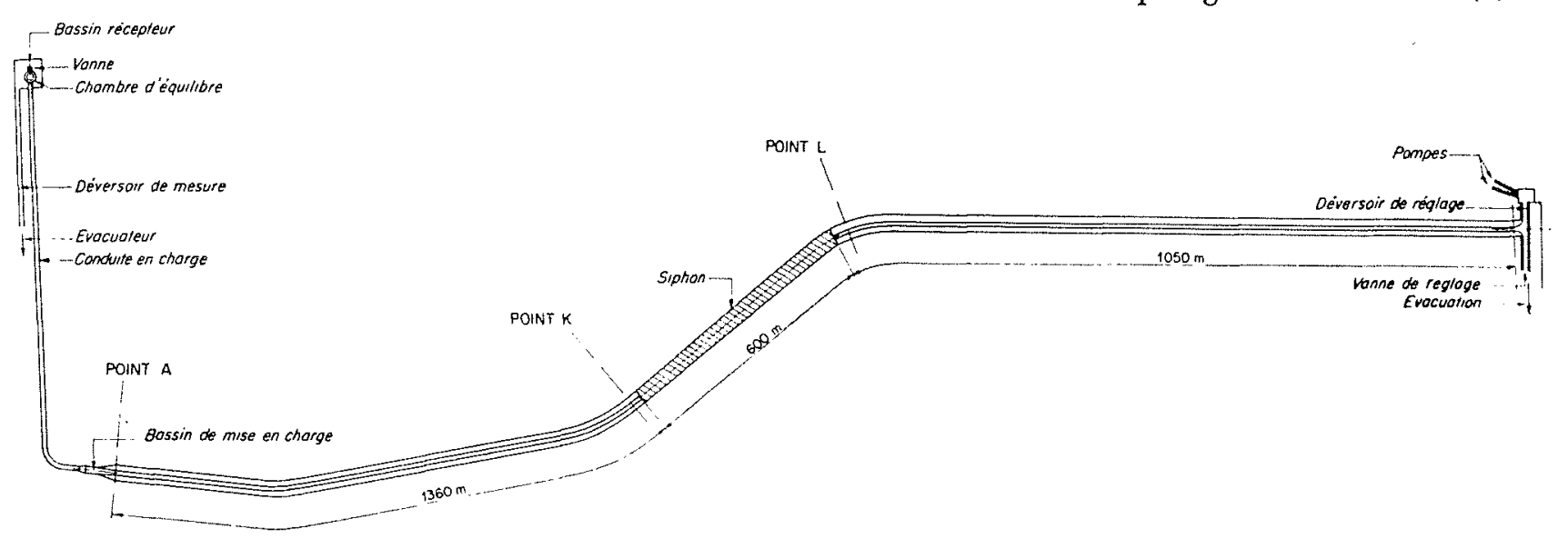

FIG. 4

Le modèle comprend, en allant de l'amont vers l'aval, toutes les dimensions étant celles de l'ouvrage réel :

- Un bassin d'alimentation;

- La première partie du canal d'amenée (longueur $1050 \mathrm{~m}$ );

- Le siphon en charge (longueur $\mathrm{L}=600 \mathrm{~m}$. Section $f=47,7 \mathrm{~m}^{2}$ );

- La deuxième partie du canal d'amenée (longueur $1360 \mathrm{~m}$ );

-. La galerie en charge (longueur $\mathrm{L}=500 \mathrm{~m}$. Section $F=38,5 \mathrm{~m}^{2}$ );

- La cheminée d'équilibre $\left(F=154 \mathrm{~m}^{2}\right)$.

Les autres données résultant des conditions d'expérimentation et servant de base à l'étude théorique sont les suivantes :

- Débit initial $Q_{0}=135 \mathrm{~m}^{3} / \mathrm{s}$.

- Tirant d'eau au bassin de mise en charge ............ 7,07 m

_. Tirant d'eau à l'aval du siphon.... . 6,98 m

- Tirant d'eau à l'amont du siphon. . $8,36 \mathrm{~m}$

- Perte de charge dans le siphon $\mathrm{P}_{0}^{\prime}=1,38 \mathrm{~m}$

Différence de cote du plan d'eau entre l'aval du siphon et le bassin de mise en charge :

$$
h=0,182 \mathrm{~m}
$$

La figure 4 reproduit les dispositions principales de l'installation.

Les débits sont mesurés à l'aide d'un déversoir type de Bazin.

Les variations du plan d'eau sont enregistrées à l'aide d'un dispositif mis au point par le Laboratoire et essentiellement constitué par deux électrodes verticales plongeant dans l'eau $\left(^{*}\right)$.
Les variations du plan d'eau sont enregistrées aux points suivants:

- Bassin de mise en charge;

- Section de sortie du siphon;

-- Section d'entrée du siphon.

Les expériences ont été eflectuées en éliminant l'influence de la cheminée d'équilibre par coupure du débit à l'amont de celle-ci.

\section{2. - Etude théorique \\ de la propagation des intumescences}

Nous considérons également, poux l'étude théorique, l'arrêt brusque total du débit $\mathrm{Q}_{0}=135 \mathrm{~m}^{3} / \mathrm{s}$, effectué à l'amont de la chambre d'équilibre.

Nous avons effectué l'étude théorique en utilisant successivement deux constructions graphiques, correspondant à des hypothèses différentes.

La première qui est, à priori, la plus rigoureuse, tient compte des pertes de charge et de la pente du fond; elle est établie avec des caractéristiques rectilignes de pente $(a \pm \mathcal{U}) / g \mathrm{~S}$, selon la méthode rappelée précédemment. L'épure con-

(*) J. Lagasse et J. Novgano: Note aux Comptes rendus Académie des Sciences, t. 234, 1952. 


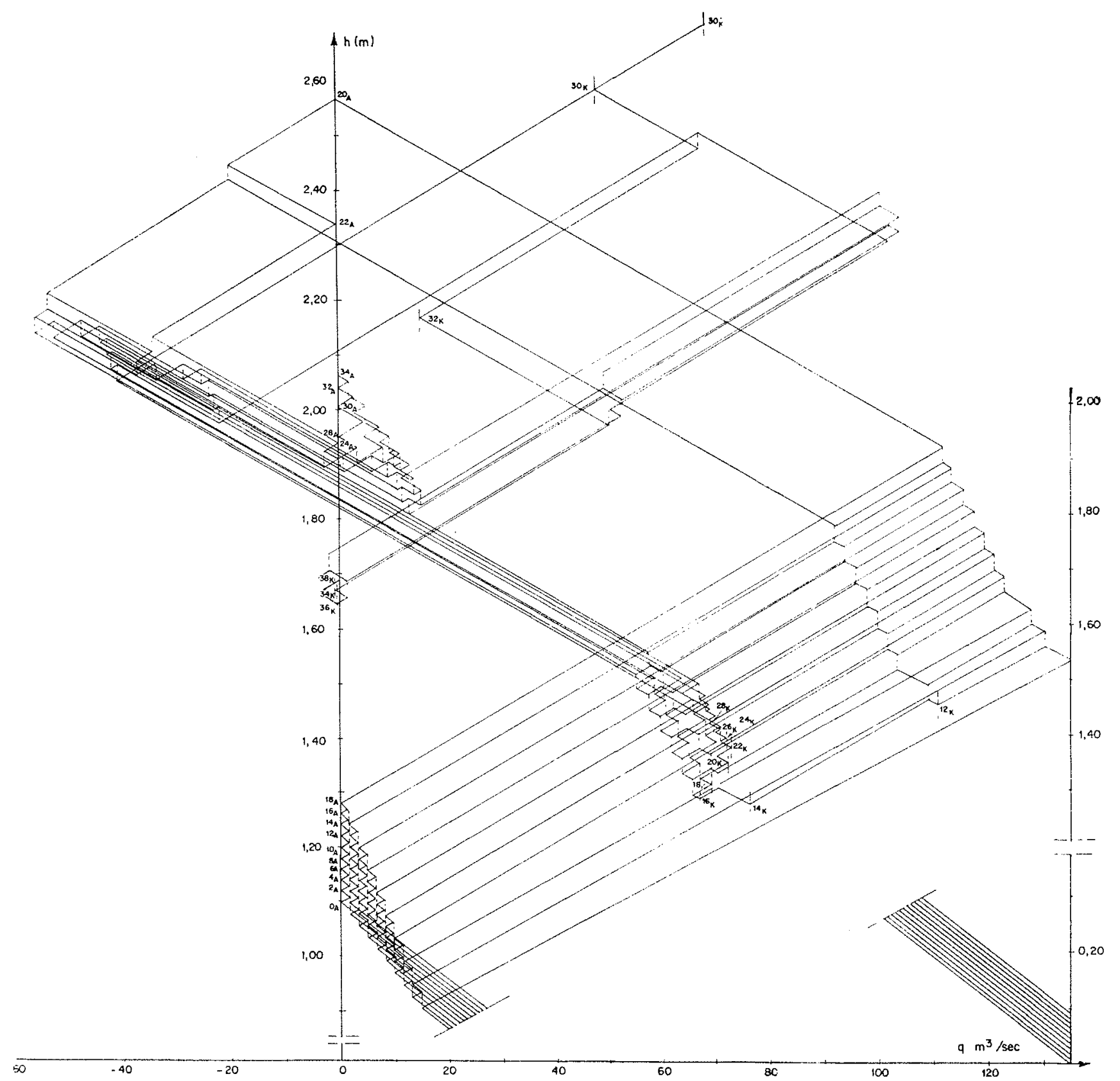

Fig. :

cernant la partic aval du canal d'amenée est reproduite sur la figure 5 .

La seconde construction graphique néglige l'influence des pertes de charge et de la pente du fond du canal : en première approximation, nous considérons le canal d'amenée comme horizontal et les conditions initiales tiennent compte $d u$ fait que la différence d'altitude de la ligne d'eau, pour la partie aval du canal d'amenée, est $h=0,182 \mathrm{~m}$. D'autre part, comme la construction graphique met en jeu des débits faibles, pour les points intermédiaires, nous négligeons l'influence des pertes de charge sur le décalage des caractéristiques rectilignes. Comme nous le verrons, les résultats obtenus et la comparaison avec les relevés effectués sur modèle justifient cette approximation. La construction graphique concernant la partie aval du canal est reproduite sur la figure 6.

Les constructions graphiques donnent le plan d'eau aux divers points du canal d'amenée.

Pour comparer les résultats théoriques avec les enregistrements effectués sur le modèle réduit, nous n'avons reproduit que les trois cour- 
bes correspondant aux points où les enregistrements ont été effectués. La figure 7 reproduit les courbes théoriques déduites de l'épure et du calcul aux différences finies dans le cas où l'on tient compte des pertes de charge et de la pente du fond d'une part, et dans le cas de l'épure simplifiée, d'autre part.

En dehors de toute comparaison avec l'expérience, le rapprochement des valeurs obtenues par les deux épures justifie l'approximation in- troduite par la construction graphique simplifiée : les différences de cote sont, au plus, égales à $0,10 \mathrm{~m}$.

Or, les constructions graphiques, quelle que soit la méthode employée, ne peuvent faire apparaître les oscillations secondaires qui accompagnent la formation d'un front d'ondes et qui sont particulièrement importantes à la réflexion du siphon; on se rend compte de la très bonne approximation que l'on obtient par la méthode

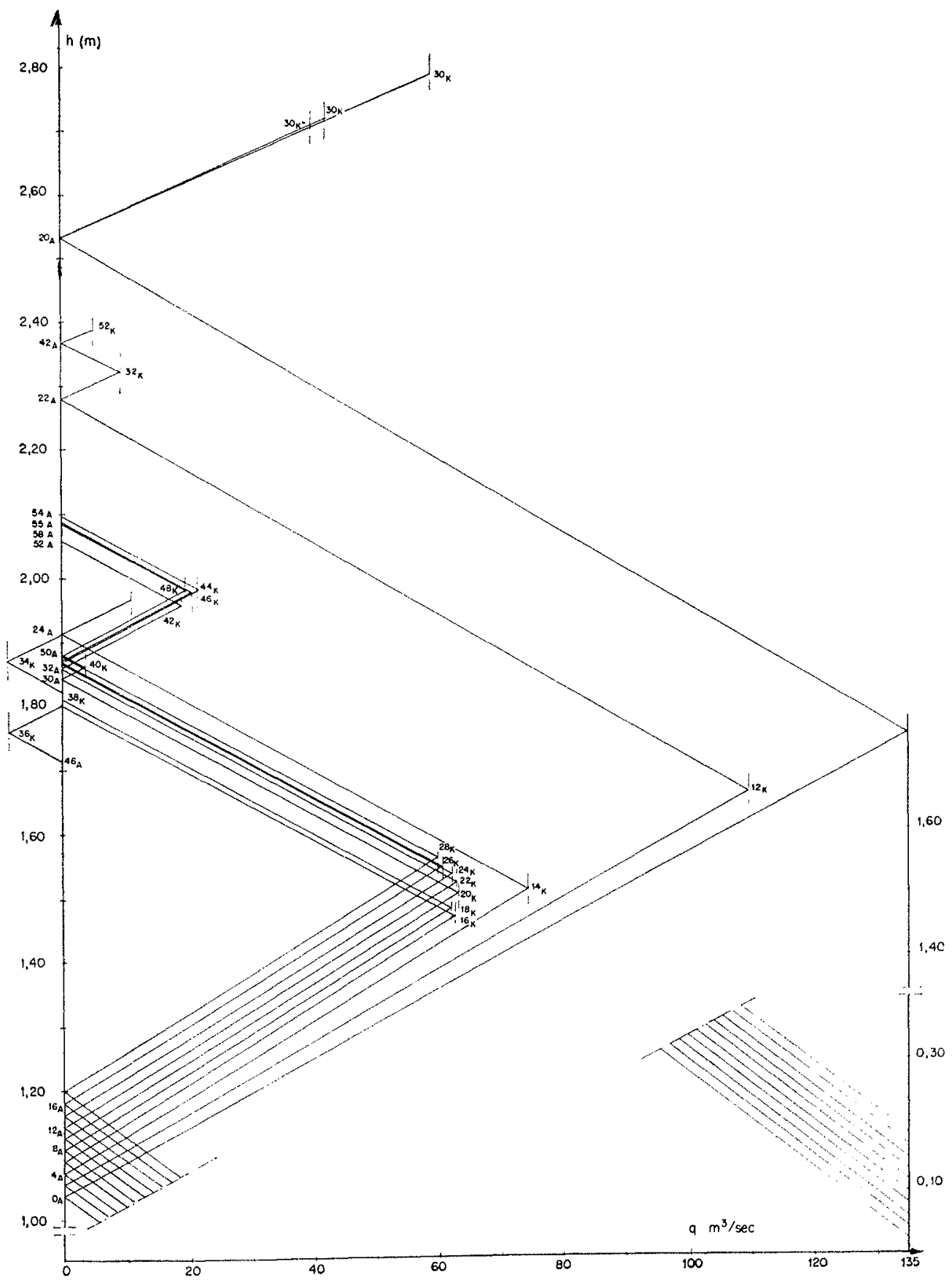

FIG. 6 


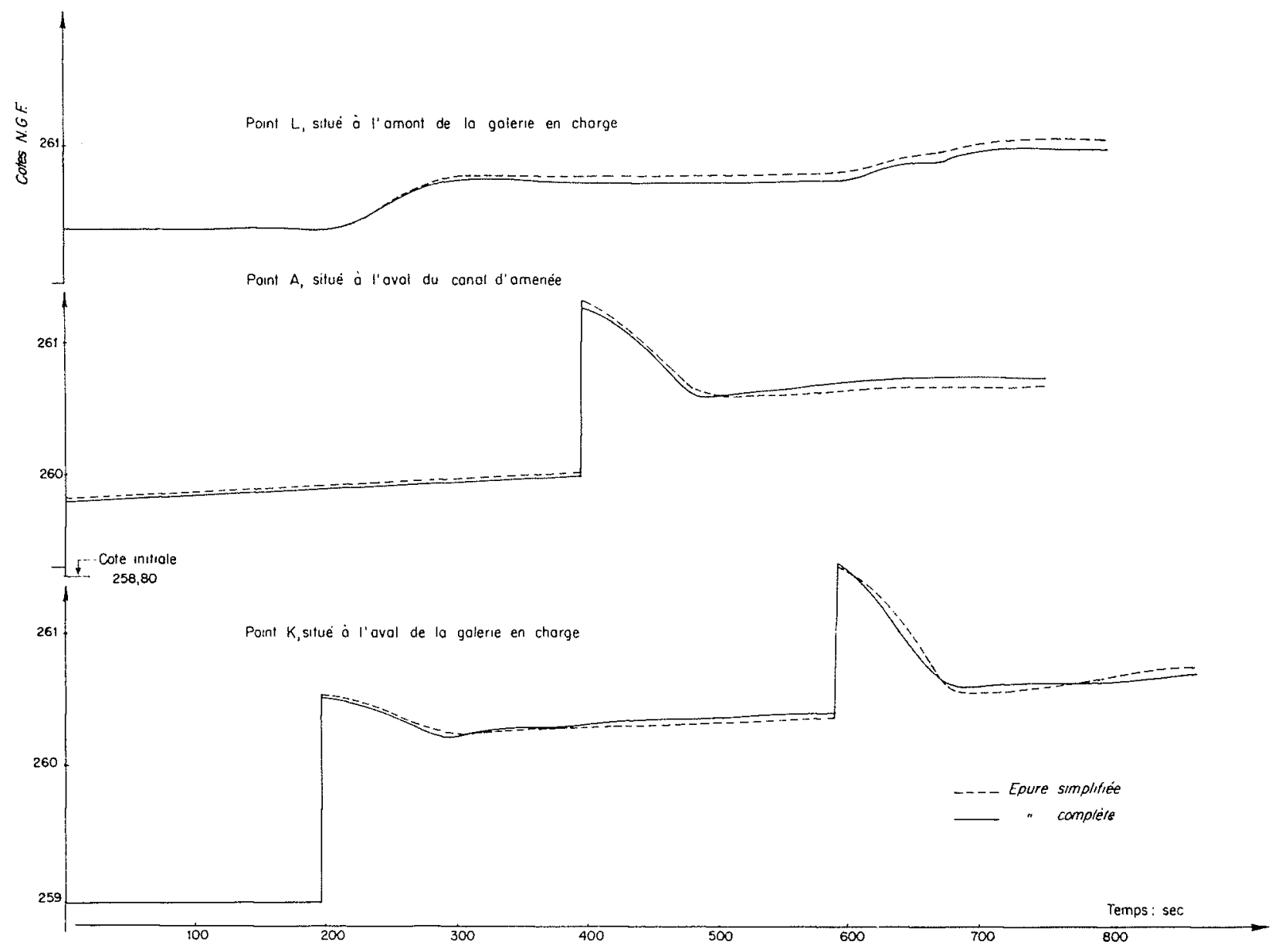

FIG. 7

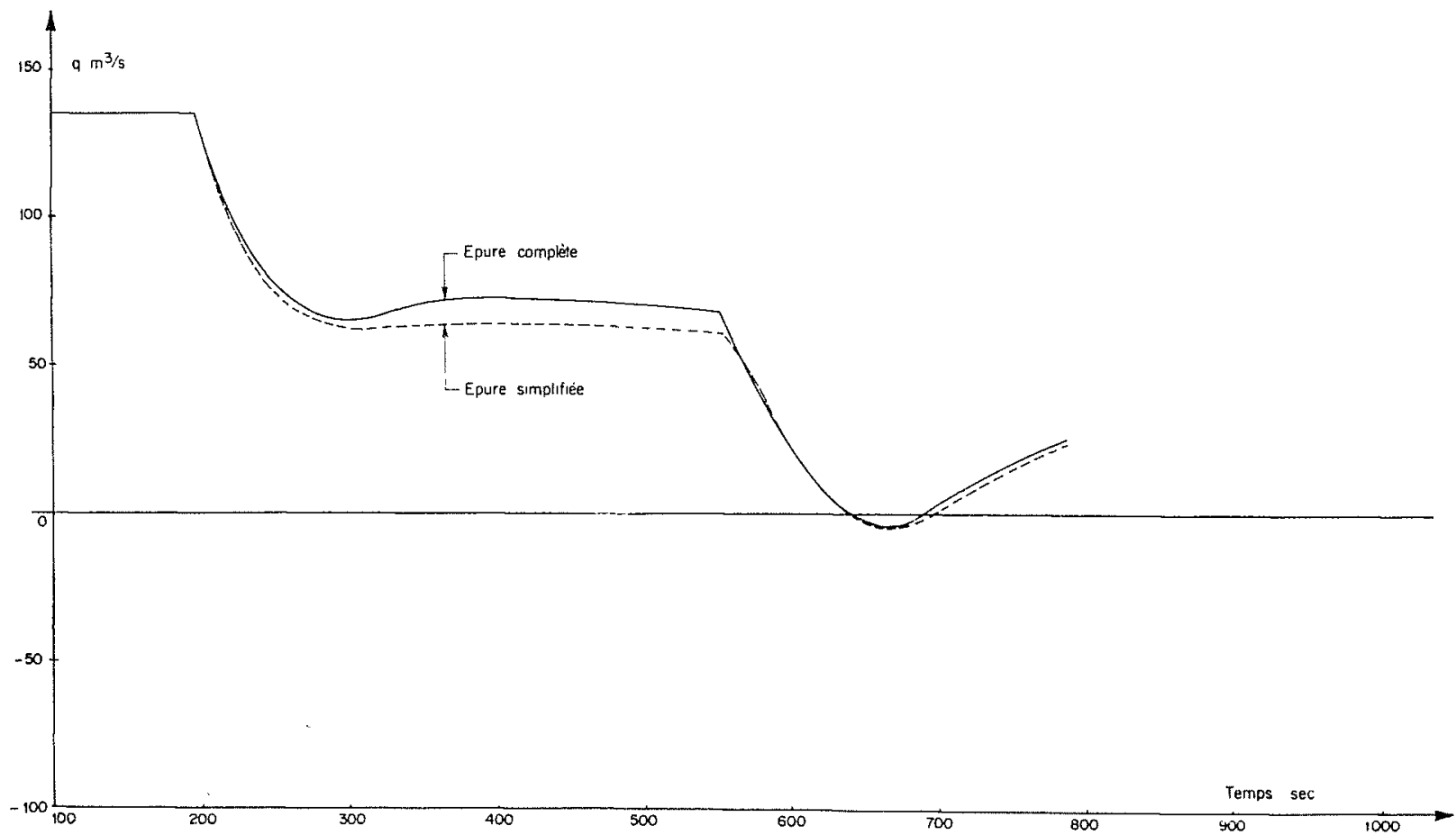

Fig. 8 
simplifiée, particulièrement intéressante par le gain notable de temps qu'elle apporte à la construction graphique.

Les constructions graphiques permettent également de déterminer la variation de débit dans le siphon, en fonction du temps. Les courbes données par la figure 8 reproduisent les variatiuns obtenues au moyen des deux constructions fournis par les deux constructions graphiques est très bonne.

\section{3. - Vérification expérimentale}

La différence entre les deux épures théoriques étant très faible, comme nous venons de le voir, nous ne retenons, pour la comparaison avec les résultats expérimentaux, que les valeurs théori-

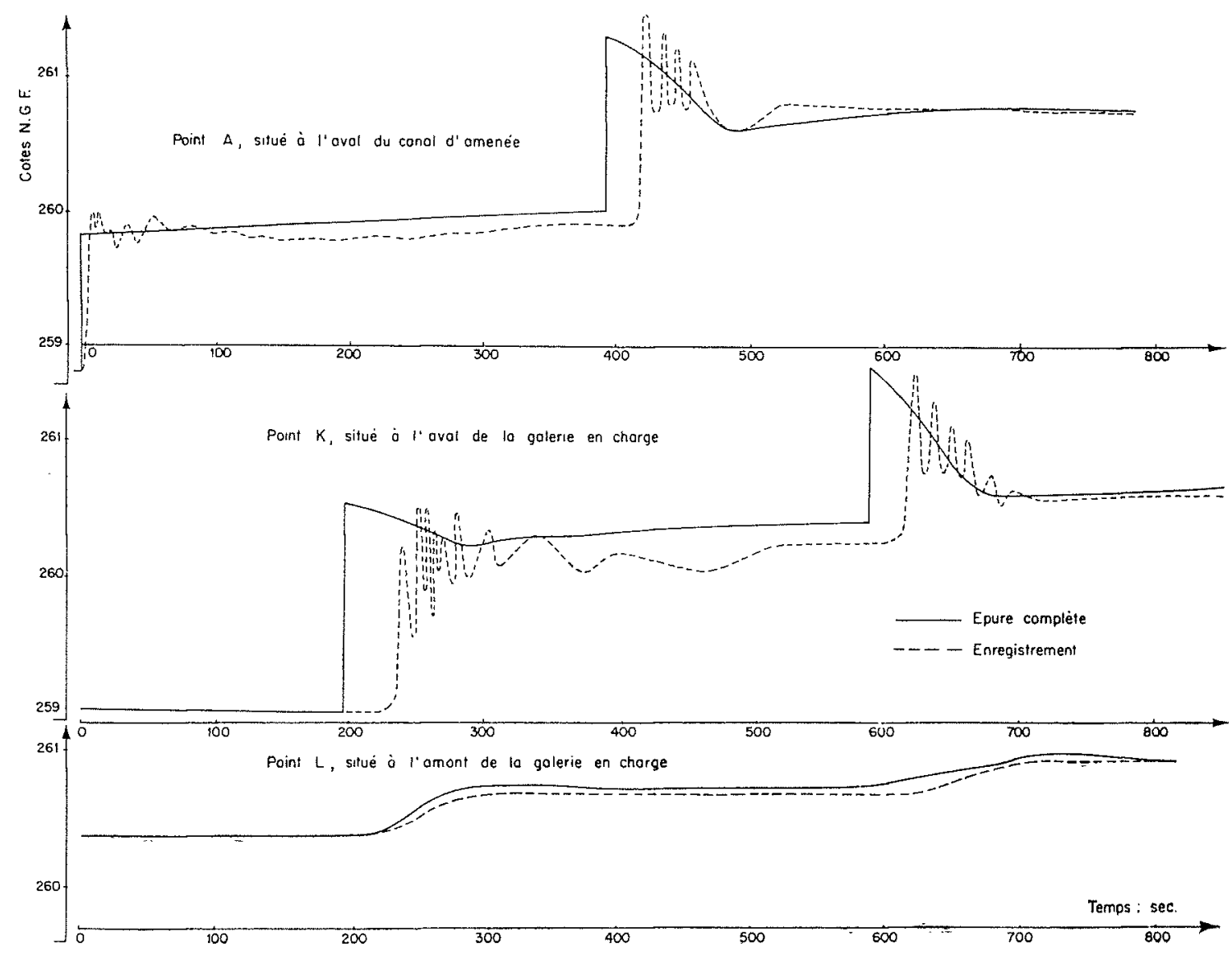

FIG. 9

graphiques. Elles montrent que le débit diminue assez rapidement jusqu'à une valeur de l'ordre de $60 \mathrm{~m}^{3} / \mathrm{s}$; celle-ci se maintient constante jusqu'au moment où l'intumescence réfléchie au bassin de mise en charge atteint le siphon. Le débit, à partir de cet instant, diminue à nouveau très vite, s'annule, puis reprend une certaine valeur avec, par la suite, des oscillations dues au fait que l'écoulement s'effectue dans le sens amont aval ou en sens inverse.

Ici encore, la concordance entre les résultats ques fournies par la méthode utilisant la première construction graphique.

Les courbes théoriques et expérimentales sont reproduites sur la figure 9 .

Les valeurs théoriques sont toujours légèrement supćrieures aux valeurs moyennes expérimentales: les écarts sont d'ailleurs très faibles et nettement inférieurs à l'amplitude des oscillations secondaires.

Les méthodes théoriques que nous avons indiquées fournissent une bonne approximation. 